\title{
Physical interpretation of frequency-modulation atomic force microscopy
}

\author{
Franz J. Giessibl and Hartmut Bielefeldt \\ Universität Augsburg, Institut für Physik, Experimentalphysik 6, D-86135 Augsburg, Germany
}

(Received 24 March 1999)

\begin{abstract}
Frequency modulation atomic force microscopy is a method for imaging the surface of metals, semiconductors and insulators in ultrahigh vacuum with true atomic resolution. The imaging signal in this technique is the frequency shift $\Delta f$ of an oscillating cantilever with eigenfrequency $f_{0}$, spring constant $k$ and amplitude $A$, which is subject to tip-sample forces $F_{t s}$. Here, we present analytical results of $\Delta f\left(f_{0}, k, A\right)$ for several basic classes of $F_{t s}$. With these results, a method to calculate images is derived and demonstrated with an example.
\end{abstract}

The scanning tunneling microscope (STM) has provided spectacular images of conducting surfaces on the atomic scale. ${ }^{1}$ After the invention of atomic force microscopy (AFM) by Binnig, Quate, and Gerber in 1986, ${ }^{2}$ the possibility of imaging insulating surfaces with atomic resolution in real space seemed to be very close. True atomic resolution with static AFM on inert samples has been reported in $1993 .^{3}$ However, imaging reactive surfaces like Si (111) in ultrahigh vacuum by static AFM has proven to be impractical because of chemical bonding between tip and sample and wear on the atomic scale. ${ }^{4}$ The application of frequency modulation AFM (FM-AFM) (Ref. 5) with large amplitudes in noncontact mode has helped to overcome problems with tip-sample bonding and finally, the $7 \times 7$ reconstruction of $\mathrm{Si}$ (111) could be resolved also by AFM. ${ }^{6-7}$ The initial experiments have been refined and the quality of FM-AFM images reaches that of the STM. ${ }^{8-10}$ Other semiconductors, ${ }^{10}$ ionic crystals, ${ }^{11}$ metal oxides, ${ }^{12}$ metals, ${ }^{13,14}$ organic monolayers, ${ }^{15}$ a film of xenon physisorbed on graphite, ${ }^{16}$ and deliberately created defects on $\mathrm{CaF}_{2}(111)$ (Ref. 17) have been imaged with atomic resolution. The first and second international workshop on noncontact AFM (NC-AFM98 in Osaka, Japan and NC-AFM99 in Pontresina, Switzerland) have helped to establish FM-AFM as a powerful experimental technique. While the number of surfaces which are imaged by FMAFM with atomic resolution is growing rapidly, open questions in the quantitative interpretation of the FM-AFM remain. The imaging signal in FM-AFM is the shift in resonance frequency $\Delta f$ of an oscillating cantilever (CL). Here, we derive for the first time analytical solutions of $\Delta f$ for three classes of tip-sample potential $V_{t s}$ : inverse power-, power- and exponential potentials. With this relations, we establish a powerful method for the calculation of FM-AFM images.

In FM-AFM, a CL with a very high- $Q$ factor $\left(\approx 10^{5}\right)$, eigenfrequency $f_{0}$ and spring constant $k$ is subject to controlled positive feedback such that it oscillates with a constant amplitude $A$. When this CL is brought close to a sample, its frequency changes from $f_{0}$ to $f=f_{0}+\Delta f$. This frequency change $\Delta f$ is used to create an image $z_{\mathrm{CL} b}(x, y, \Delta f)$ by scanning the $\mathrm{CL}$ in the $x-y$ plane while adjusting the $z$ position of the base of the $\mathrm{CL}_{\mathrm{CL}_{-} b}$ such that $\Delta f$ stays constant (see Fig. 1). Typical parameters are $\Delta f$ $\approx-100 \mathrm{~Hz} k \approx 20 \mathrm{~N} / \mathrm{m}, f_{0} \approx 200 \mathrm{kHz}$ and $A \approx 10 \mathrm{~nm}-$ see Table I in Ref. 18 for an overview.

The frequency shift $\Delta f$ as a function of $F_{t s}=-\partial V_{t s} / \partial z$ has been calculated by first order perturbation theory using the Hamilton-Jacobi approach ${ }^{19}$

$$
\Delta f\left(\mathbf{x}_{\mathrm{CL}_{-} b}, f_{0}, k, A\right)=-\frac{f_{0}^{2}}{k A^{2}} \int_{0}^{1 / f_{0}} F_{t s}\left(\mathbf{x}_{\mathrm{tip}}\right) q^{\prime}(t) d t
$$

with the CL deflection $q^{\prime}(t)=A \cos \left(2 \pi f_{0} t\right)$ and $\mathbf{x}_{\text {tip }}$ $=\left[x_{\mathrm{CL}_{-} b}+\text { const., } y_{\mathrm{CL}_{-} b}, z_{\mathrm{CL}_{-} b}+q^{\prime}(t)\right]^{T}$. This result has been confirmed by other approaches. ${ }^{20-25}$ First-order perturbation theory yields excellent results because $\max \left|V_{t s}\right|$ $\lesssim 10 \mathrm{eV}$, while $E=0.5 \mathrm{kA} \mathrm{A}^{2} \approx 5 \mathrm{keV}$. A Fourier approach $q^{\prime}(t)=\sum_{m=0}^{\infty} a_{m} \cos (2 \pi m f t)$ (Refs. 20-22) shows that both the de deflection $\left(a_{0}=A \Delta f / f_{0}\right)$ and the higher orders are very small $\left[\left|a_{m}\right| \leqslant 2 A /\left(1-m^{2}\right)\left|\Delta f / f_{0}\right|\right.$ for $m \geqslant 2$ with $\Delta f / f_{0} \lesssim 10^{-3}$ ] compared to the fundamental amplitude $a_{1}$ $=A$. At the lower turnaround point of the tip $\mathbf{x}_{\mathrm{tip}}^{\mathrm{ltp}}$ the precise value of the deflection of the CL is given by the condition of constant energy $E=0.5 k q_{\text {ltp }}^{\prime 2}+V_{t s}\left(\mathbf{x}_{\text {tip }}^{\text {ltp }}\right)=0.5 k A^{2}$, thus $q_{\text {ltp }}^{\prime} \approx$ $-A+A \cdot V_{t s}\left(\mathbf{x}_{\text {tip }}^{\mathrm{ltp}}\right) / 2 E$. Since $\left|V_{t s}\right|$ is small compared to $E$, the FM-AFM image $z_{\mathrm{CL}_{-} b}(x, y, \Delta f)$ is approximately equal to the map described by $\mathbf{x}_{\text {tip }}^{\text {ltp }}$.

The scaling properties of $\Delta f$ are such that it is useful to introduce a "normalized frequency shift", 19

$$
\gamma(z, A):=\frac{k A^{3 / 2}}{f_{0}} \Delta f\left(z, f_{0}, k, A\right) .
$$

Substituting $z^{\prime}=A\left[1+\cos \left(2 \pi f_{0} t\right)\right]$ in Eq. (1) we find

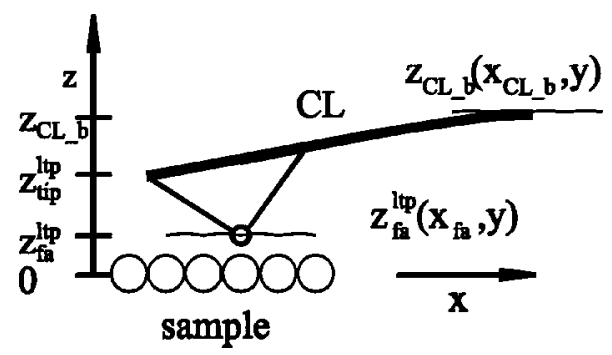

FIG. 1. Schematic of an oscillating cantilever (CL) with tip and front atom (fa) at its lower turnaround point (ltp) close to a sample. 


$$
\gamma\left(\mathbf{x}_{\mathrm{tip}}^{\mathrm{ltp}}, A\right)=\frac{1}{\sqrt{2} \pi} \int_{0}^{2 A} \frac{F_{t s}\left(\mathbf{x}_{\mathrm{tip}}^{\mathrm{ltp}}+z^{\prime} \mathbf{e}_{z}\right)}{\sqrt{z^{\prime}}} \frac{1-\frac{z^{\prime}}{A}}{\sqrt{1-\frac{z^{\prime}}{2 A}}} d z^{\prime} .
$$

In typical experiments, $A$ is very large compared to the range of $F_{t s}$ and the second factor in the integral is close to unity in regions where $F_{t s}$ is nonvanishing. Therefore, the "largeamplitude", normalized frequency shift

$$
\gamma_{l A}\left(\mathbf{x}_{\mathrm{tip}}^{\mathrm{ltp}}\right)=\int_{0}^{\infty} \frac{F_{t s}\left(\mathbf{x}_{\mathrm{tip}}^{\mathrm{ltp}}+z^{\prime} \mathbf{e}_{z}\right)}{\pi \sqrt{2 z^{\prime}}} d z^{\prime}
$$

is a good approximation for $\gamma$ for any class of tip-sample forces, provided that the range of the forces is small compared to $A$.

The interaction of a macroscopic tip of an AFM with a sample is a complicated many-body problem, which can be solved with molecular dynamics calculations. ${ }^{26}$ For a qualitative analysis, quite realistic model forces can be constructed from a chemical contribution modeling the interaction of the front atom (fa) with the sample $F_{\text {fa }}$ plus the van der Waals (vdW) contribution of the rest of the tip $F_{\text {background }}{ }^{19}$ Since $\gamma$ is linear in $F_{t s}$ it is practical to expand $F_{t s}$ in terms of basic types: (a) inverse-power forces, (b) power forces, and (c) exponential forces and superimpose the individual solutions for $\gamma$.

(a) $F_{t s}(z)=C / z^{n}$ (Lennard-Jones potential, vdW forces, electrostatic and magnetic forces ${ }^{27}$ ). Insertion in Eq. (3) and comparing the result with the integral representation of the hypergeometric function $F_{c}^{a, b}(\mathrm{~s})$ (Ref. 28) yields:

$$
\gamma(z, A)=\frac{C \sqrt{A}}{z^{n}}\left[F_{1}^{n, 0.5}\left(\frac{-2 A}{z}\right)-F_{2}^{n, 1.5}\left(\frac{-2 A}{z}\right)\right] .
$$

For large amplitudes $(A / z \gg 1)$ the transformation formula $F_{c}^{a, b}(\varsigma)=(1-\varsigma)^{-b} F_{c}^{b, c-a}(\varsigma /(\varsigma-1))$ is useful (15.5.9. in Ref. 28) and

$$
\gamma_{l A}(z)=\sqrt{\frac{1}{2 \pi}} \frac{\Gamma\left(n-\frac{1}{2}\right)}{\Gamma(n)} \frac{C}{z^{n-1 / 2}}
$$

where $\Gamma(n)$ is the Gamma function. ${ }^{28}$

TABLE I. Three basic types of tip-sample potentials and corresponding normalized frequency shift $\gamma$.

\begin{tabular}{lccc}
\hline \hline Basic type & $V_{t s}(z)$ & $F_{t s} \sqrt{V_{t s} / F_{t s}}$ & $\gamma_{l A}$ \\
\hline $\begin{array}{l}\text { Inv.-power } \\
n>1, z>0\end{array}$ & $\frac{1}{n-1} \frac{C}{z^{n-1}}$ & $\frac{1}{\sqrt{n-1}} \frac{C}{z^{n-0.5}}$ & $\frac{\Gamma\left(n-\frac{1}{2}\right)}{\sqrt{2 \pi} \Gamma(n)} \frac{C}{z^{n-0.5}}$ \\
$\begin{array}{l}\text { Power } \\
m \geqslant 0, z<0\end{array}$ & $\frac{C(-z)^{m+1}}{m+1}$ & $\frac{C(-z)^{m+0.5}}{\sqrt{m+1}}$ & $\frac{\Gamma(m+1) C(-z)^{m+0.5}}{\sqrt{2 \pi} \Gamma(m+1.5)}$ \\
Exponential & $\frac{F_{0} e^{-\kappa z}}{\kappa}$ & $\frac{F_{0} e^{-\kappa z}}{\sqrt{\kappa}}$ & $\frac{F_{0} e^{-\kappa z}}{\sqrt{2 \pi \kappa}}$
\end{tabular}

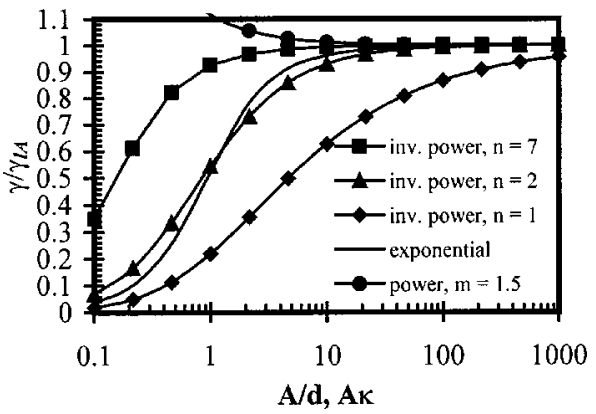

FIG. 2. Ratio between normalized frequency shift $\gamma$ and large-amplitude approximation $\gamma_{l A}$ as a function of $A / z$ and $A \kappa$, respectively.

(b) $F_{t s}(z)=C(-z)^{m}$ for $z<0$ and $F_{t s}(z)=0$ for $z>0$ (Hertzian contact forces with $m=3 / 2$ for a spherical tip on a flat surface and adhesion forces with $m=0$ ) (Ref. 27)

$\gamma=\frac{C|z|^{m+1 / 2} \Gamma(m+1)}{\sqrt{2 \pi}}\left[\frac{F_{m+1.5}^{0.5,0.5}\left(\frac{-z}{2 A}\right)}{\Gamma(m+3 / 2)}+\frac{z}{2 A} \frac{F_{m+2.5}^{0.5,1.5}\left(\frac{-z}{2 A}\right)}{\Gamma(m+5 / 2)}\right]$.

For large amplitudes

$$
\gamma_{l A}(z)=\frac{\Gamma(m+1)}{\sqrt{2 \pi} \Gamma(m+3 / 2)} C(-z)^{m+1 / 2} \text { for } z<0 .
$$

(c) $F_{t s}(z)=F_{0} e^{-\kappa z}$ (Morse potential)

$$
\gamma=F_{0} e^{-\kappa z} \sqrt{A}\left[M_{1}^{0.5}(-2 \kappa A)-M_{2}^{1.5}(-2 \kappa A)\right],
$$

where $M_{b}^{a}(\mathrm{~s})$ is the Kummer function. ${ }^{28}$ For large amplitudes $(\kappa A \gg 1)$ we can use the asymptotic expression of $M_{b}^{a}(\mathrm{~s})$ (13.5.1. in Ref. 28) and find

$$
\gamma_{l A}(z)=\frac{F_{0} e^{-z \kappa}}{\sqrt{2 \pi \kappa}} .
$$

On conductive samples, simultaneous STM and FM-AFM operation is possible. Since the bandwidth of the tunneling current $\left(I_{t}\right)$ preamplifier is i.g. much smaller than $f_{0}$ of the $\mathrm{CL}$, the measured $I_{t}$ is a time-average. With $I_{t}(z)=I_{0} e^{-\kappa_{t} z}$ we find

$$
\left\langle I_{t}(z, A)\right\rangle=I_{0} e^{-\kappa_{t} z} M_{1}^{1 / 2}\left(-2 \kappa_{t} A\right) .
$$

When $\kappa_{t} A \gg 1,\left\langle I_{t}(z, A)\right\rangle / I_{t}(z, 0) \approx 1 / \sqrt{2 \pi \kappa_{t} A}$ yielding a reduction to $1 / 35$ for typical parameters $\left(A=10 \mathrm{~nm}\right.$ and $\kappa_{t}$ $\approx 20 \mathrm{~nm}^{-1}$ for metals).

Figure 2 shows the ratio $\gamma(z, A) / \gamma_{l A}(z)$ as a function of $A / z$ and $A \kappa$, respectively. In a typical experiment, $A / z$ and $A \kappa$ are between 10 and 1000 during imaging where $\gamma_{l A}(z)$ and $\gamma(z, A)$ are almost identical. As $A / z$ becomes smaller, the sensitivity to chemical (short range) forces increases. In addition to a better signal-to-noise ratio ${ }^{18}$ the use of smaller amplitudes makes sharp tips less important since the sensitivity to the macroscopic vdW forces decreases.

Equation (4) shows that $\gamma_{l A}$ increases with the range of $F_{t s}$ and $\gamma$ has the dimension of $\mathrm{N} \sqrt{\mathrm{m}}$. Table I shows $\gamma_{l A}$ and $F_{t s} \sqrt{\lambda}$ with range $\lambda$ for three basic types of forces laws. For exponential forces $F_{t s}(z)=F_{0} e^{-\kappa z}$ the range $\lambda$ defined by 


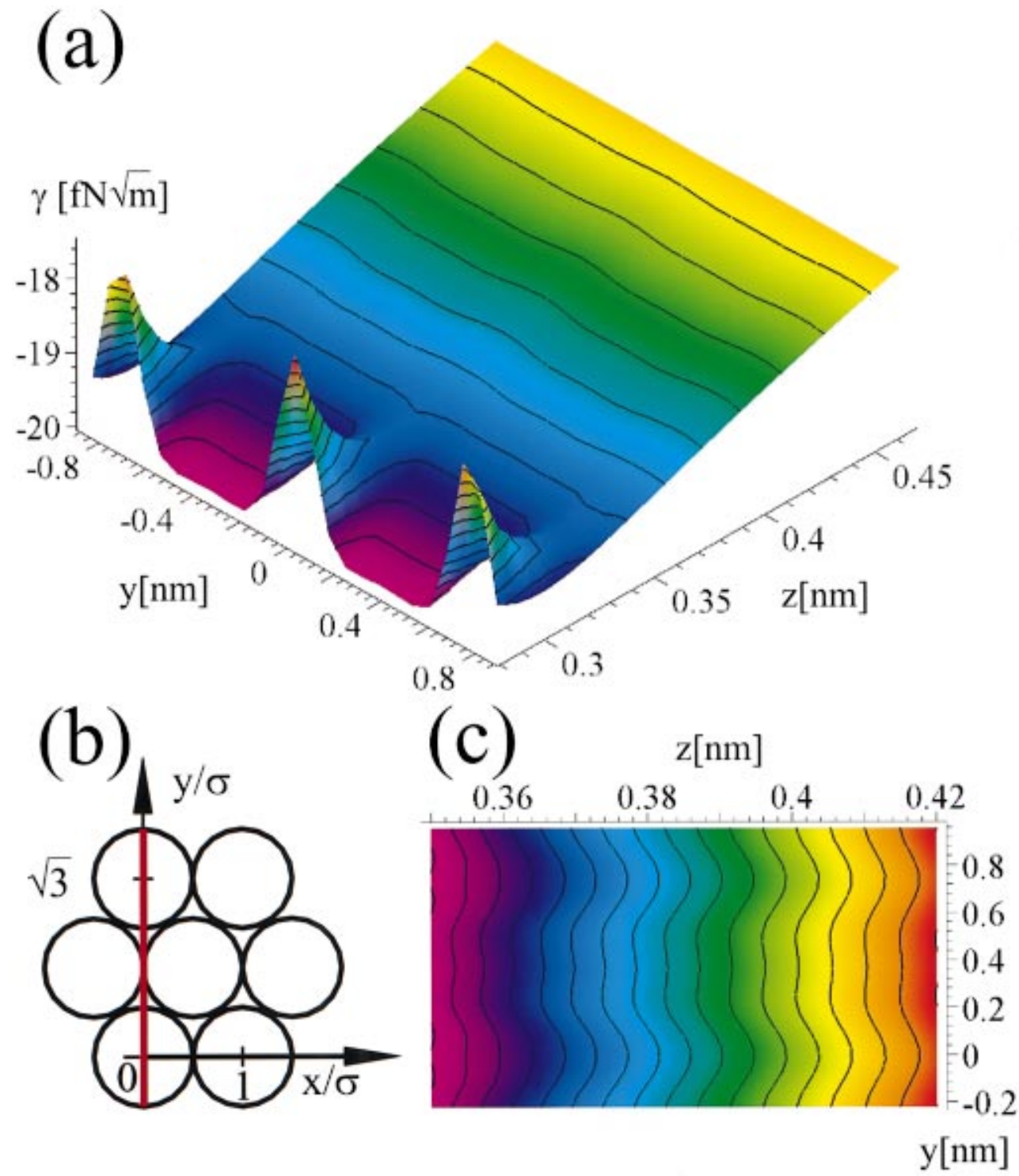

FIG. 3. (Color) (a) Normalized frequency shift $\gamma_{I A}(x=0, y, z)$ from $y=-2 \sigma$ to $2 \sigma(\sigma=0.43 \mathrm{~nm})$. Xenon atoms are situated at $y=0, \pm \sqrt{3} \sigma$. The contour lines of constant $\gamma$ are cross sections of the corresponding FM-AFM image $z(x, y, \gamma)$. Maximal corrugation is obtained for $\gamma \approx-18 \mathrm{fN} \sqrt{\mathrm{m}}$ (green area). (b) Arrangement of Xenon atoms on the surface, (c) top view of $\gamma_{l A}$ along red track in (b) showing a maximal corrugation of $\sim 5 \mathrm{pm}$.

$V(z+\lambda) / V(z)=1 / e$ is independent of $z$ with $\lambda=V(z) / F(z)$ $=1 / \kappa$. For inverse power and power forces, an analog expression $\lambda_{\text {power }}:=V(z) / F(z)$ depends on $z$, but it shows ana$\log$ scaling properties, i.e., $V\left(z+\lambda_{\text {power }}\right) / V(z) \approx 1 / e$ for exponents $n>1 \quad(=1 / e$ for $n \rightarrow \infty)$. Table I shows $\gamma_{l A}$ and $F_{t s} \sqrt{V_{t s} / F_{t s}}$. It is interesting to note that $F_{t s} \sqrt{V_{t s} / F_{t s}} / \gamma_{l A}$ $\approx \sqrt{2 \pi}$ for $|n| \geqslant 2$ and $F_{t s} \sqrt{V_{t s} / F_{t s}} / \gamma_{l A}=\sqrt{2 \pi}$ for exponential forces and power forces with $|n| \rightarrow \infty$. Since the frequency shift is a linear function of $F_{t s}$ [Eq. (1)], the total $\gamma$ is a linear combination of the contributions of the basic types $F_{t s}^{i}$

$$
\gamma_{l A}\left(\mathbf{x}_{\mathrm{tip}}^{\mathrm{ltp}}\right) \approx \frac{1}{\sqrt{2 \pi}} \sum_{i} F_{t s}^{i}\left(\mathbf{x}_{\mathrm{tip}}^{\mathrm{ltp}}\right) \sqrt{V_{t s}^{i}\left(\mathbf{x}_{\mathrm{tip}}^{\mathrm{ltp}}\right) / F_{t s}^{i}\left(\mathbf{x}_{\mathrm{tip}}^{\mathrm{ltp}}\right)}
$$

where $V_{t s}^{i}$ is a basic type. Typical chemical forces are $\approx-1$ $\mathrm{nN}$ with a range of $0.1 \mathrm{~nm}$ and $\mathrm{vdW}$ forces are $\approx-1 \mathrm{nN}$ with a "range" of $1 \mathrm{~nm}$, thus experimental $\gamma^{\prime} s$ are expected to be in the order of $-10 \mathrm{fN} \sqrt{\mathrm{m}}$.
To illustrate how Eq. (12) can be used to calculate the FM-AFM image $z(x, y, \gamma)$, we consider a relatively simple system: an adsorbed layer of xenon. Xenon forms a closed packed film with next-neighbor distance $\sigma_{\mathrm{Xe}} \approx 0.43 \mathrm{~nm}$ on graphite, thus the unit vectors of the xenon surface lattice are given by $\mathbf{a}_{1}=(\sigma, 0,0)^{T}$ and $\mathbf{a}_{2}=(\sigma / 2, \sigma \sqrt{3} / 2,0)^{T}$. Allers et al. ${ }^{16}$ have succeeded in imaging such a layer by FM-AFM: the image shows the expected closed packed structure with a corrugation of $25 \mathrm{pm}$ and atoms appearing high in the image, i.e. no contrast inversion. The following imaging parameters have been used: $\Delta f=-92 \mathrm{~Hz}, k=35 \mathrm{~N} / \mathrm{m}, f_{0}=160 \mathrm{kHz}$ and $A=9.4 \mathrm{~nm}$, i.e., at $\gamma=-18 \mathrm{fN} \sqrt{\mathrm{m}}$.

The interaction of two xenon atoms can be modeled by a Lennard Jones potential

$$
\phi_{\mathrm{LJ}}(d)=-E_{\mathrm{bond}}\left[2\left(\frac{\sigma}{d}\right)^{6}-\left(\frac{\sigma}{d}\right)^{12}\right]
$$

with $E_{\text {bond }}=0.02 \mathrm{eV}$ and $\sigma=0.433 \mathrm{~nm} \cdot{ }^{29}$ Since the tip was made of silicon, we assume that the front atom of the tip is 
also silicon. Krupp has shown, that the Hamaker constant of two materials is given by the geometric average between the individual Hamaker constants. ${ }^{30}$ With further assuming, that the equilibrium distance in a silicon-xenon bond is given by the mean value between the bulk nearest neighbor distance in $\mathrm{Si}\left(\sigma_{\mathrm{Si}}=0.235 \mathrm{~nm}\right)$ and Xe, we can create a LJ model potential for interaction of $\mathrm{Si}$ and $\mathrm{Xe} \phi_{\mathrm{LJ}}^{\mathrm{Si}-\mathrm{Xe}}$ with $E_{\text {bond }}^{\mathrm{Si}-\mathrm{Xe}}$ $=0.047 \mathrm{eV}$ and $\sigma_{\mathrm{Si}-\mathrm{Xe}}=0.33 \mathrm{~nm} . F_{t s}$ is approximated by:

$$
F_{t s}\left(\mathbf{x}_{\mathrm{fa}}^{\mathrm{ltp}}\right)=-\frac{C}{z_{\mathrm{fa}}^{\operatorname{ltp}}+\sigma_{\mathrm{Si}}}+\frac{\partial}{\partial z} \sum_{n, m=-\infty}^{\infty} \phi_{\mathrm{LJ}}^{\mathrm{Si}-\mathrm{Xe}}\left(\left|\mathbf{x}_{\mathrm{fa}}^{\mathrm{ltp}}-\mathbf{a}_{m}^{n}\right|\right),
$$

where $C$ is a constant (the tip is assumed to be conical or pyramidal, thus the long-range component is given by $-C / z$, see Refs. 19 and 31) and $\mathbf{a}_{m}^{n}=n \mathbf{a}_{1}+m \mathbf{a}_{2}$. For the calculation of $\gamma_{l A}(x, y, z)$, the attractive part $\phi_{\mathrm{LJ}-\mathrm{att}} \propto d^{-6}$ and repulsive part $\phi_{\mathrm{LJ}-\mathrm{rep}} \propto d^{-12}$ have to be treated separately. ${ }^{32}$ Figure 3(a) shows $\gamma_{l A}(x=0, y, z)$ from $y$ $=-2 \sigma$ to $y=2 \sigma$. The contour lines correspond to cross sections of the image $z(x, y=0, \gamma=$ const. $)$. Stable operation of the microscope is only possible in a $z$ range where $\partial \gamma / \partial z$ $<0$. The maximal corrugation occurs at $\gamma_{\text {optimal }}$ $\approx-18 \mathrm{fN} \sqrt{\mathrm{m}}$ and is $\approx 5 \mathrm{pm}$ [Fig. 3(c)]. It is noted that the absolute value of $\gamma_{\text {optimal }}$ depends strongly on $C$ (here $C$ $=8.6 \times 10^{-19} \mathrm{~J}$ ) which is a function of the macroscopic tip shape. However, the maximal corrugation varies little with $C$ and is quite insensitive to the parameters we have calculated for the front atom-sample potential $\phi_{\mathrm{LJ}}^{\mathrm{Si}-\mathrm{Xe}}$. The deviation between the corrugations in theory and experiment is probably due to elastic sample deformation. We assume that the tip is rigid (i.e., $\mathbf{x}_{\mathrm{fa}}-\mathbf{x}_{\mathrm{tip}}$ is constant-the force constant be- tween next neighbors in $\mathrm{Si}$ is $170 \mathrm{~N} / \mathrm{m}$ ), but the vdW bonds in the Xe are weak $\left(\partial^{2} \phi_{\mathrm{LJ}} / \partial d^{2} \approx 1 \mathrm{~N} / \mathrm{m}\right)$. We believe that the corrugation is strongly enhanced because the $\mathrm{Xe}$ atoms are pulled out of the surface. A similar effect has been observed in STM where the theoretical corrugation for $\mathrm{Al}(111)$ was $\sim 1 \mathrm{pm}$ while the experimental values were $\sim 50 \mathrm{pm}^{33}$

In summary, we have found a physical interpretation of large amplitude FM-AFM: the images are a map $z(x, y, \gamma$ = const. $)$ where $\gamma(x, y, z)=\int_{0}^{\infty} F_{t s}\left(x, y, z+z^{\prime}\right) / \sqrt{2 \pi^{2} z^{\prime}} d z^{\prime}$. When $F_{t s}$ is known in terms of basic (i.e., power, inverse power, exponential) force types $F_{t s}^{i}(z), \quad \gamma$ $=\Sigma_{i} F_{t s}^{i}(z) \sqrt{\lambda_{i} / 2 \pi}$ where $\lambda_{i}=V_{t s}^{i}(z) / F_{t s}^{i}(z)$. Our analytic results for various basic types of tip-sample forces establish the validity of the large-amplitude approximation for the interpretation of images and allow a quantitative analysis of $\Delta f(z)$ curves for larger $z$ values (i.e., $0<A / z<100$ ). We have further found an analytic result for the dependence of the tunneling current as a function of amplitude. Calculations with molecular dynamics, which currently yield $F_{t s}(x, y, z)$ (Ref. 26) can be extended to compute the observables in FM-AFM, namely the experimental FM-AFM images $z(x, y, \gamma)$ with Eq. (4) and $\gamma(x, y, z, A)$ curves with Eq. (3) on specific spots $x, y$ on the sample (e.g. adatom sites and cornerhole centers). Comparing the experimental results with these calculations, the force vs distance characteristics of chemical bonds between front atom and samples can be directly derived.

We thank A. Baratoff, P. van Dongen, U. Dürig, S. Hembacher, U. Mair, and J. Mannhart for discussions and W. Allers et al. for the preprint of Ref. 16. This work is supported by the BMBF (Project No. 13N6918/1).
${ }^{1}$ G. Binnig et al., Phys. Rev. Lett. 49, 57 (1982); 50, 120 (1983).

${ }^{2}$ G. Binnig et al., Phys. Rev. Lett. 56, 930 (1986).

${ }^{3}$ F. Ohnesorge and G. Binnig, Science 260, 1451 (1993).

${ }^{4}$ L. Howald et al., Z. Phys. B: Condens. Matter 93, 267 (1994).

${ }^{5}$ T. R. Albrecht et al., J. Appl. Phys. 69, 668 (1991).

${ }^{6}$ F. J. Giessibl, Science 267, 68 (1995); S. Kitamura and M. Iwatsuki, Jpn. J. Appl. Phys., Part 2 34, L145 (1995).

${ }^{7}$ P. Güthner, J. Vac. Sci. Technol. B 14, 2428 (1996).

${ }^{8}$ R. Lüthi et al., Z. Phys. B: Condens. Matter 100, 165 (1996).

${ }^{9}$ R. Erlandsson et al., Phys. Rev. B 54, R8309 (1996).

${ }^{10}$ Y. Sugawara et al., Science 270, 1648 (1995).

${ }^{11}$ M. Bammerlin et al., Probe Microsc. 1, 3 (1997).

${ }^{12}$ K. Fukui et al., Phys. Rev. Lett. 79, 4202 (1997); H. Raza et al., ibid. 82, 5265 (1999).

${ }^{13}$ Ch. Loppacher et al., Appl. Surf. Sci. 140, 287 (1999).

${ }^{14}$ S. Orisaka et al., Appl. Surf. Sci. 140, 243 (1999).

${ }^{15}$ B. Gotsmann et al., Eur. Phys. J. B 4, 267 (1998).

${ }^{16}$ W. Allers et al., Europhys. Lett. 48, 276 (1999).

${ }^{17}$ M. Reichling and C. Barth, Phys. Rev. Lett. 83, 768 (1999).

${ }^{18}$ F. J. Giessibl et al., Appl. Surf. Sci. 140, 352 (1999).
${ }^{19}$ F. J. Giessibl, Phys. Rev. B 56, 16010 (1997).

${ }^{20}$ A. Baratoff (unpublished).

${ }^{21}$ A. I. Livshits et al., Phys. Rev. B 59, 2436 (1999).

${ }^{22}$ U. Dürig, Surf. Interface Anal. 27, 467 (1999).

${ }^{23}$ H. Hölscher et al., Appl. Surf. Sci. 140, 344 (1999).

${ }^{24}$ N. Sasaki and M. Tsukada, Jpn. J. Appl. Phys., Part 2 37, L533 (1998); Appl. Surf. Sci. 140, 339 (1999).

${ }^{25}$ L. Wang, Appl. Phys. Lett. 73, 3781 (1998).

${ }^{26}$ R. Perez et al., Phys. Rev. Lett. 78, 10835 (1997).

${ }^{27}$ J. Israelachvili, Intermolecular and Surface Forces, 2nd ed. (Academic, London, 1991).

${ }^{28}$ M. Abramowitz and I. Stegun, Handbook of Mathematical Functions, 9th ed. (Dover, New York, 1970).

${ }^{29}$ N. Ashcroft and N. D. Mervin, Solid State Physics (Saunders College, Philadelphia, 1981), p. 398.

${ }^{30}$ H. Krupp, Adv. Colloid Interface Sci. 1, 113 (1967).

${ }^{31}$ U. Hartmann, J. Vac. Sci. Technol. B 9, 465 (1991).

${ }^{32}$ For $\phi(d)=C / d^{n}$ with $d=\sqrt{x^{2}+y^{2}+z^{2}}, \partial \phi / \partial z \neq-N C / d^{n+1}$ if $x, y \neq 0$ but the deviation is negligible for $z \geq x, y$.

${ }^{33}$ J. Winterlin et al., Phys. Rev. Lett. 62, 59 (1989). 